\title{
Recycling assessment and Test validation of Constant velocity drive shaft for Santana Automobile
}

\author{
$\mathrm{LU} X 1^{1, a^{*}}, \mathrm{ZUO} \mathrm{LI}^{1, \mathrm{~b}}$
}

${ }^{1}$ College of Mechanical Engineering,University for Science and Technology,Shanghai

aluxi_usst@163.com, bzuo13817315166usst@163.com

Keywords: Drive shaft;Natural frequency;Surface Hardness;Residual strength;Residual life Abstract. Using constant velocity universal drive shaft of Santana Automobile for different years as specimen,mechanical characteristics such as natural frequency and hardness were investigated.The results show that natural frequency of drive shaft decreases slightly with the increase of years. The maximum of decreased-percentage for natural frequency is $1.2 \%$. The high-rank natural frequency decreases more obvious than the low-rank, but no regulation was found.Surface hardness of different cross-sections change slightly, which shows a trend from rise to decline.According to the changes of natural frequency and hardness, it predicts that drive shafts have over $60 \%$ service life.

\section{Introduction}

The amount of automobile in Chinese increases constantly,up to154million at the end of 2014. Meanwhile,recycling problem of automobile is becoming more and more significant.In recent 10 years, State has staged a series of positive policy on recycling and reuse of automobile.Parts of scraped automobile will be reused considering enough residual life,with the ability to enter the next operating cycle.Consequently, it appears more increasingly important to assess parts of scraped automobile ${ }^{[1]}$.

Recycling assessment technology abroad on scraped automobile parts is increasingly perfectly. Our country still hasn't mastered the core technology of recycling assessment so far, currently only relying on experience and simple wear such as method of capacitance,metal magnetic memory testing and acoustic emission to predict residual life of engine and transmission. Method of capacitance was used to measure the wear of cylinder in the whole life of operation ${ }^{[2]}$.Acoustic emission diagnosis technology was used to test surface for each cylinder of engine ${ }^{[3]}$. Method of metal magnetic was taken to find out macroscopic defects on the block of engine ${ }^{[4]}$.These methods didn't put the fatigue damage, residual strength and residual life together,hard to predict residual strength and residual life of typical mechanical fatigue with no wear.Lu ${ }^{[5]}$ summed up that natural frequency of transmission gear begins to decrease with the development of fatigue damage and keeps decreasing. $\mathrm{Li}^{\left[{ }^{[6]}\right.}$ concluded that change of natural frequency for welding joint can be used to predict residual life. Ye ${ }^{[7]}$ found that change of surface hardness is divided into rising, stable and falling three stages. Sandor $\mathrm{BI}^{[8]}$ utilized that change of hardness can be used to estimate the fatigue strength and residual life in the process of high cycle fatigue damage of medium carbon steel. These results provide reference for evaluating fatigue damage and predicting residual strength and residual life of drive shaft.

Using shafts as specimen,the change of the typical mechanical characteristic such as the natural frequency and surface hardness were studied.It is initially revealed that drive shaft used for 20 years is in the top $60 \%$ of the fatigue life. Through static strength test,evaluation were validated.The results provide practical data and technical reference for assessing the recycling of the drive shaft with the application of typical mechanical properties such as natural frequency and hardness. 


\section{Materials and methods}

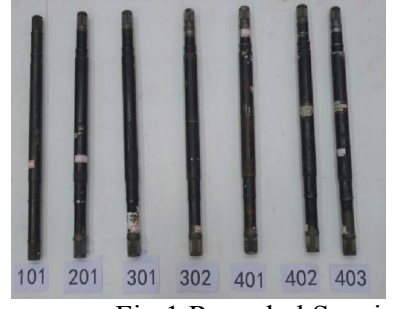

Fig.1 Recycled Specimen

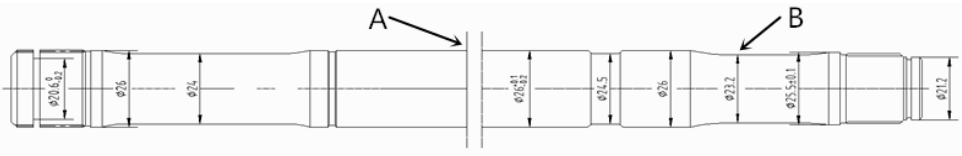

Fig.2 Sketch of measuring position for hardness-test

Material of recycled drive shaft of Santana automobile is $40 \mathrm{Cr}$, which treated by quenching \& heat tempering. The range of hardness is $52 \mathrm{HRC} \sim 58 \mathrm{HRC}$, with the hardening layer over $3 \mathrm{~mm}$. Samples are shown in figure1,101was never used,201was used for 10 years,301 and302 were used for 15 years, 401,402 and 403 were used for 20years.

Research methods and steps are as follows:

First.Visual inspection.All the samples were separated,cleaned,classified and marked,then magnetic flaw detection and appearance check were made to eliminate samples which have obvious cracks,injury-bending and bruises.

Second.Modal test.Using the method of hammer,a single acceleration sensor was chosen to pick up the signal in order to reduce the influence on the dynamic characteristics from sensor and its cable. Drive shaft was dived into ten parts, the acceleration sensors were put on the second point.Other points were knocked by hammer one after one,each point was repeated for five times, taking the average.

Third.Modal analysis and calculation.Using DASP system to fit the measured data to get the first four rank natural frequency.

Fourth.Hardness test.Considering shaft bears torque in operation and its structure size,we select two locations where diameter is in maximum and minimum to measure the hardness (shown in figure2).Diameter of section A is in maximum while diameter of section B is in minimum.Each section willed be measured four times at circumferential direction, the final hardness value is the average of four times.

Fifth.Static strength test.The static strength test was carried out on drive shafts which were used for 20 years to obtain data for static strength.Starting the machine,commissioning;Clamping the drive shafts,testing and then recording.

Modal test was conducted by DASP system of Beijing Oriental Institute of vibration \& noise technology.The Sensor used in the test is8776A50M3accelerometer produced by Kistler with the measuring range from-500g to $+500 \mathrm{~g}$.Hardness test was conducted on theHRS150digital Rockwell Hardness Tester.The test force dwell time is $5 \mathrm{~s}$, and the load is $150 \mathrm{~kg}$.HRC hardness scale was chosen to operate the hardness test.The sites of modal test and hardness test are shown in figure3and4.The NZ-10000 static torsion testers shown in figure 5were chosen to operate the strength test,maximum torque of which is $50000 \mathrm{~N} \cdot \mathrm{m}$. The torque was applied at a constant speed of $180 \mathrm{r} / \mathrm{min}$.

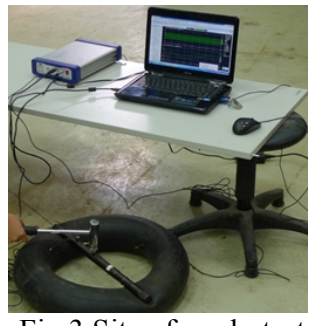

Fig.3 Site of mode-test

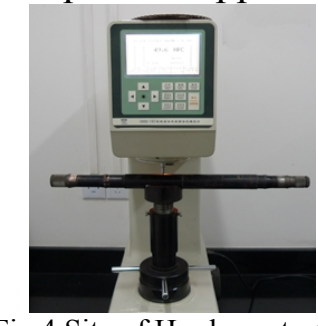

Fig.4 Site of Hardness-test

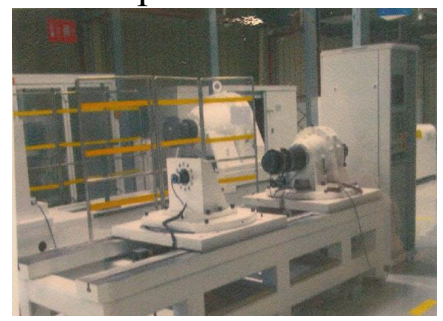

Fig.5 Testing machine of static strength

\section{Experimental results and analysis}

\section{Modal test}

Applying the method of hammer to knock the drive shaft one point after one,we obtain the first four rank natural frequency of each sample by using DASP system to fit and analyze the measured data. We recorded the data of samples with the same service life in average which is shown in table1. 
Tab.1 The first-four-rank natural frequency of specimen

\begin{tabular}{|c|c|c|c|c|c|c|c|c|c|}
\hline Number & Service-life & Rank1 & Mean & Rank2 & Mean & Rank3 & Mean & Rank4 & Mean \\
\hline 101 & 0 & 549.8 & 549.8 & 1380.6 & 1380.6 & 2542.5 & 2542.5 & 4028.1 & 4028.1 \\
\hline 201 & 10 & 546.3 & 546.3 & 1368.4 & 1368.4 & 2524.4 & 2524.4 & 4011.1 & 4011.1 \\
\hline 301 & & 545.9 & & 1365.6 & & 2517.9 & & 4003.4 & \\
\hline 302 & 15 & 546.3 & 546.1 & 1365.4 & 1365.5 & 2518.2 & 2518.1 & 3998.3 & 4000.9 \\
\hline 401 & & 545.1 & & 1363.9 & & 2515.5 & & 3993.2 & \\
\hline 402 & 20 & 545.3 & 545.1 & 1364.8 & 1364.4 & 2516.9 & 2517.1 & 3993.7 & 3994.4 \\
\hline 403 & & 544.9 & & 1364.6 & & 2518.9 & & 3996.3 & \\
\hline
\end{tabular}

To visually reflect the natural frequency with change of service life,we draw up the figure of natural frequency for different years which is shown in figure 6,according to the data in table 1 .

By the test results:

The first-rank natural frequency of specimen which was used for 10 years reduces $3.5 \mathrm{~Hz}$ relative to sample 101 ,falling by $0.64 \%$.The second-rank natural frequency reduces $12.2 \mathrm{~Hz}$ relative to sample 101 ,falling by $0.88 \%$. The third-rank natural frequency reduces $18.1 \mathrm{~Hz}$ relative to sample 101 , falling by $0.71 \%$. The fourth-rank natural frequency reduces $17 \mathrm{~Hz}$ relative to sample 101 , falling by $0.42 \%$.

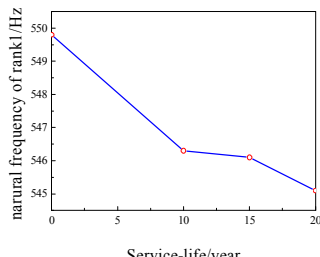

(a)

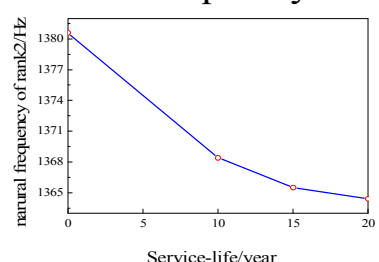

(b)

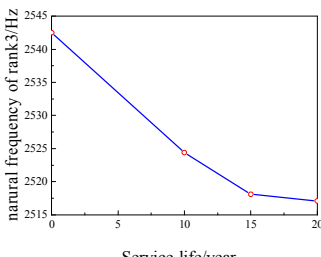

(c)

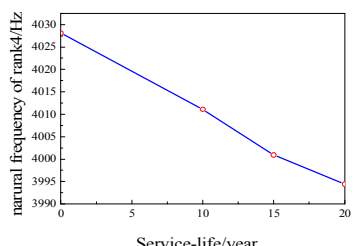

(d)

Fig.6 Natural frequency for different years of specimen

The first-rank natural frequency of specimen which was used for 15 years reduces $3.7 \mathrm{~Hz}$ relative to sample 101 ,falling by $0.67 \%$.The second-rank natural frequency reduces $15.1 \mathrm{~Hz}$ relative to sample 101 ,falling by $1.10 \%$.The third-rank natural frequency reduces $24.4 \mathrm{~Hz}$ relative to sample101,falling by $0.96 \%$. The fourth-rank natural frequency reduces $27.2 \mathrm{~Hz}$ relative to sample101, falling by $0.68 \%$.

The first-rank natural frequency of specimen which was used for 20 years reduces $4.7 \mathrm{~Hz}$ relative to sample 101 ,falling by $0.85 \%$. The second-rank natural frequency reduces $16.2 \mathrm{~Hz}$ relative to sample 101 ,falling by $1.17 \%$. The third-rank natural frequency reduces $25.4 \mathrm{~Hz}$ relative to sample101, falling by $1.0 \%$. The fourth-rank natural frequency reduces $33.7 \mathrm{~Hz}$ relative to sample101, falling by $0.84 \%$.

The first four rank natural frequency of samples decrease slightly relative to 101 along with the increase of service life.The high order drops greater than the low order in the change of the natural frequency,but no regulation was found.The maximum of decreased-percentage for natural frequency is $1.2 \%$. We consider that the first four rank natural frequency of all the samples never change in the operation.

Literature ${ }^{[6]}$ lists the relationship between the fatigue life and the dropping-rate of natural frequency for the spot welding.Natural frequency of the spot welding never change in the top $40 \%$ of fatigue life,dropping obviously in $70 \%-80 \%$ of fatigue life.In the last $20 \%-30 \%$ of fatigue life,natural frequency drops sharply.In the top $40 \%$ of fatigue life,drive shaft suffers tiny damage with the strength remaining unchanged.We consider that all the drive shaft chosen for the test have over $60 \%$ remaining life and are rich in residual strength. When designed,the strength of shafts could be reduced appropriately.Drive shafts which used for 20 years still have the value of recycling.

\section{Hardness test}

Hardness test was conducted according to requirements of the test gradually. We recorded the surface hardness of samples with the same service life in average which is shown in table2. 
Tab. 2 Hardness of specimen for different years

\begin{tabular}{ccccc}
\hline Measuring position & Number & Measurement & Hardness & Mean \\
\hline \multirow{3}{*}{ Position A } & 101 & $50.3 、 53.8 、 54.0 、 54.7$ & 53.2 & 53.2 \\
& 201 & $56.0 、 53.6 、 55.3 、 54.6$ & 54.9 & 54.9 \\
& 301 & $55.0 、 52.6 、 52.7 、 57.3$ & 54.4 & 54.4 \\
& 302 & $54.2 、 55.2 、 55.6 、 52.2$ & 54.3 & \\
& 401 & $50.0 、 48.9 、 56.6 、 48.7$ & 50.3 & \\
& 402 & $52.7 、 52.6 、 52.0 、 54.9$ & 53.0 & 52.1 \\
& 403 & $50.3 、 53.2 、 53.4 、 54.5$ & 52.9 & \\
Position B & 101 & $54.7 、 51.5 、 49.7 、 53.7$ & 52.4 & 52.4 \\
& 201 & $52.3 、 55.6 、 53.6 、 56.4$ & 54.5 & 54.5 \\
& 301 & $54.4 、 55.6 、 55.6 、 52.2$ & 54.5 & 53.5 \\
& 302 & $54.5 、 50.4 、 52.5 、 52.2$ & 52.4 & \\
& 401 & $56.4 、 55.3 、 49.5 、 52.8$ & 53.5 & \\
& 402 & $54.0 、 52.4 、 53.1 、 54.2$ & 53.4 & 52.9 \\
& 403 & $50.7 、 53.1 、 51.8 、 51.4$ & 51.8 & \\
\hline
\end{tabular}

[HRC]

By the test results:

Surface hardness of drive shafts changes slightly at the base of the original hardness on average with the increase of service life,but changes very little.Surface hardness in the maximum and minimum stress location both have a trend of decline after rising. The minimum value is $52.1 \mathrm{HRC}$ and the maximum is54.9HRC,still in the design requirements within the scope of (52HRC-58HRC). Surface hardness in the maximum stress location presents a increasing trend ranging from 0 to 10 years.Surface hardness in the maximum stress location presents a decreasing trend ranging from 10 to 20 years,with the largest dropping rate from 15 to 20 years. The average surface hardness of the maximum stress location of drive shaft which used for 20 years is smaller than the original hardness.

Surface hardness in the minimum stress location presents a increasing trend ranging from 0 to 10 years.Surface hardness in the minimum stress location presents a decreasing trend ranging from 10 to 20 years,with the smallest dropping rate from 15 to 20 years. The average surface hardness of the minimum stress location of drive shaft which used for 20 years is bigger than the original hardness mainly because of no or miner damage in the process of operation.

Literature $^{[7]}$ gives the relationship between the change of hardness and dropping percentage of fatigue life for the medium carbon steel.Surface hardness presents a obvious trend of increase in the early fatigue life( $0-10 \%$ ).In 20\%-30\% of fatigue life,surface hardness begins to decrease, gradually falling to the lowest.

In the top $30 \%$ stage of fatigue life,drive shaft suffers miner fatigue damage (damage smaller than 0.15).It deduces that strength remains unchanged and sufficient in residual strength with over $70 \%$ remaining life in the future.Because of richness in residual strength,strength of drive shaft can be appropriately reduced when designing.Drive shafts are still valuable after being used for 20 years.

\section{Static-strength test}

By studying the change of natural frequency and surface hardness of drive shaft for different years,it takes a preliminary infer that drive shafts which used for 20 years are still sufficient in residual life and strength.Static strength test were followed to explore whether the drive shafts have the ability to meet the requirements in operation after the fatigue service for a long time.Table 3 lists the test results of static strength for drive shaft.

Table 3 Results of static strength test for drive shaft

\begin{tabular}{cccc}
\hline Number & Test Condition & Residual Strength $(\mathrm{N} \cdot \mathrm{m})$ & Torsional Stiffness $\left(\mathrm{N} \cdot \mathrm{m} /{ }^{\circ}\right)$ \\
\hline 403 & $0^{\circ}$ & 4061 & 148.5 \\
404 & $0^{\circ}$ & 3847 & 140.7 \\
405 & angled & 3911 & 146.5 \\
406 & angled & 4013 & 145.2 \\
\hline
\end{tabular}

Based on the regulation of metal material in the process of fatigue damage, the residual strength remains basely when recycle ratio is less than 0.6,dropping at the reach of 0.9.Considering the test results and the degradation model for residual strength of metal material,drive shafts are still sufficient in residual strength at the reach of scrap stage. 


\section{Conclusions}

According to the test results, we achieve the following conclusion:

(1)Top four rank natural frequency of all drive shafts decreased with the increasing of service life.Amplitude of decline is minor.The maximum of decreased-percentage for natural frequency is $1.2 \%$. The high rank of natural frequency in dropping is greater than the low rank, but no regulation was found.In the top $40 \%$ of fatigue life,drive shaft suffers tiny damage with the strength remaining unchanged.We consider that all the drive shaft chosen for the test have over $60 \%$ remaining life and are rich in residual strength.

(2)Surface hardness of different sections changes slightly relative to 101, but changes very little.Surface hardness in the maximum and minimum stress location both have a trend of decline after rising. The minimum value is $52.1 \mathrm{HRC}$ and the maximum is $54.9 \mathrm{HRC}$,still in the requirements of designing within the scope of (52HRC 58HRC). The average surface hardness of the maximum stress location of drive shaft which used for 20 years is smaller than the original hardness. The average surface hardness of the minimum stress location of drive shaft which used for 20 years is bigger than the original hardness mainly because of no or miner damage in the process of operation.

(3)Based on the regulation of metal material in the process of fatigue damage,the residual strength remains basely when recycle ratio is less than 0.6,dropping at the reach of 0.9.Considering the test results and the degradation model for residual strength of metal material,drive shafts are still sufficient in residual strength at the reach of scrap stage.

(4)According to the change of natural frequency and hardness, we consider that drive shaft which used for 20 years suffer minor damage,have over $60 \%$ remaining life and are rich in residual strength. When designed,the strength of shafts could be reduced appropriately.Drive shafts which used for 20 years still have the value of recycling.

(5)The change of natural frequency and hardness with the increasing of service life obtained from modal and hardness test is not obvious.Mechanical characteristic parameters should be considered comprehensively such as natural frequency and hardness when evaluating the assessment of drive shafts.

\section{References}

[1]YU JunWei.Analysis of scrap car recycling and reuse[J].Quality and Standardization,2011(6): 28-31.

[2]ZHANG Xilin,HE Lifeng.Wear Detection and Life Prediction of Cylinder Liner for Internal Combustion Engine[J].VEHICLE ENGINE,2008,2(1):81-84.

[3]Zhang Laibin, Yang Zhancai,Wang chaohui.Experimental study on engine piston-liner wear fault diagnosis by acoustic emission technology[J].Journal of the University of Petroleum(Natural Science Edition),2001,25(5):60-62.

[4]Liu Li, Xu Bin, Zhang Jingqiu.Study on Application of Metal Magnetic Memory Method for Nondestructive Testing of Diesel Engine Block[J].Diesel Engine,2011,33(6):39-41.

[5]LU Xi,Zheng SongLin,LI PingPing. The Change of inherent frequency of gears in the course of reinforcement and damage[J]Chinese Journal of Mechanical Engineering.2008,19(9):1087-1090.

[6]Li Chengshan,Shang Deguang,Wang Ruijie.Fatigue Life Prediction Based on Dynamic Response Characteristics for Spot-welded Joints under Variable Amplitude Loading[J].Journal of the Mechanical Engineering ,2009,45(4):70-74.

[7]YE DuYi,Wang Dejun,PING An.The experimental study on the characteristic of the surface micro-hardness for medium carbon steel during high-cycle fatigue[J].Journal of Mechanical Strength,1996(6)18(2):63-65.

[8]Sandor BI.Fundamentals of cyclic stress and strain.Madison(WI):University of Wisconsin Press, 1972. 